\title{
Originals
}

\section{Medial arterial calcification and its association with mortality and complications of diabetes}

\author{
J.E. Everhart, D.J.Pettitt, W.C. Knowler, F. A. Rose and P.H. Bennett \\ Diabetes and Arthritis Epidemiology Section, National Institute of Diabetes and Digestive and Kidney Diseases, Phoenix, Arizona, USA
}

\begin{abstract}
Summary. Medial arterial calcification was studied among 4,553 subjects in a 20-year, longitudinal study of Pima Indians. The prevalence and incidence of medial arterial calcification were highest among men, the elderly, and patients with Type 2 (non-insulin-dependent) diabetes mellitus. Medial arterial calcification was most commonly observed in the feet and appeared to progress proximally. Proportional hazards analysis was used to evaluate risk factors for medial arterial calcification in the feet and to evaluate medial arterial calcification as a risk factor for death and for complications of diabetes. Among diabetic patients, risk factors for medial arterial calcification were impaired vibration perception, long duration of diabetes, and high plasma glucose concentration $(p<0.01$ for each). Among nondiabetic subjects, age, male gender $(p<0.01$ for each), and high serum cholesterol concentration $(p=0.02)$ were risk factors for medial arterial
\end{abstract}

calcification. Nondiabetic subjects with medial arterial calcification did not have higher mortality rates than subjects without medial arterial calcification (rate ratio $=0.95,95 \%$ confidence interval $=0.7-1.3$ ). Diabetic patients with medial arterial calcification, compared with diabetic patients without medial arterial calcification, had 1.5 -fold the mortality rate $(95 \%$ confidence interval $=1.0-2.1), 5.5$-fold the rate of amputations $(95 \%$ confidence interval $=2.1-14.1), 2.4$-fold the rate of proteinuria ( $95 \%$ confidence interval $=1.3-4.5$ ), 1.7 -fold the rate of retinopathy ( $95 \%$ confidence inter$\mathrm{val}=0.98-2.8$ ), and 1.6 -fold the rate of coronary artery disease $(95 \%$ confidence interval $=0.48-5.4)$.

Key words: Medial arterial calcification, diabetic complications, epidemiology, incidence, prevalence.
Medial arterial calcification (MAC), also known as Mönckeberg's arteriosclerosis, is a focal calcification of the media, particularly of medium-sized arteries, that is readily and frequently identified outlining arteries on radiographs of the extremities (Fig.1). The calcium deposition in MAC is entirely within the medial layer of the arterial wall between smooth muscle cells [1]. MAC is linear in character and involves the vessel wall uniformly, resembling a ring in vessel cross-section and a railroad track in longitudinal section on radiographs [2]. Unlike intimal arterial calcification, from which it is easily discriminated, MAC has been thought by some investigators to have little or no clinical implications $[1,3]$, while others have linked it to sympathetic denervation and peripheral neuropathy [4-7]. MAC has been found commonly among men, the elderly, and those with diabetes, renal failure, or familial amyloidosis with polyneuropathy [8-12]. It has been unclear whether MAC begins as a proximal or distal arterial lesion $[8,12,13]$.

In this study, we determined the prevalence and incidence of MAC among diabetic and nondiabetic Pi- ma Indians, the anatomic progression of MAC, the effect of peripheral neuropathy and other factors on development of MAC, and the value of MAC as a predictor of mortality and diabetic complications.

\section{Subjects and methods}

\section{Subjects and measurements}

A longitudinal study of diabetes among Pima Indian residents of the Gila River Indian Community of Arizona has been in progress since 1965. This population has a very high incidence and prevalence of Type 2 (non-insulin-dependent) diabetes mellitus [14]. The present study includes data collected between February 1965 and 1 October 1985. Each community resident over 5 years of age was asked to have an examination approximately every 2 years, but only information on participatnts who were at least 15 years of age at some point during the data collection has been used in this study. Recruitment and examination were performed for research purposes and without regard for the participants' symptoms or state of health. The potential of follow-up bias for the detection of diabetes in this survey has been previously examined and no significant differences were found in the incidence of diabetes between subjects lost to study follow-up and regular survey participants [14]. All participants underwent the 


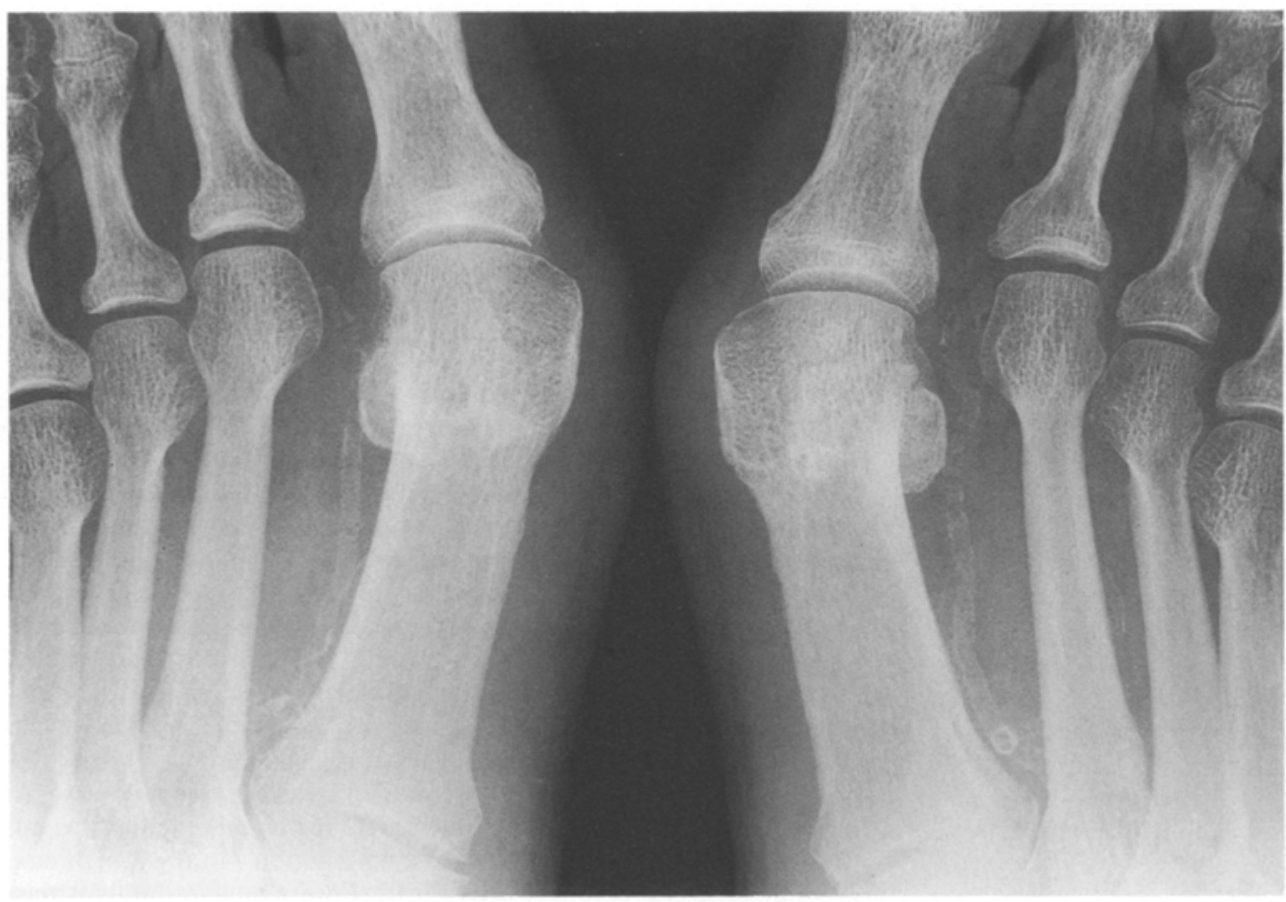

Fig. 1. Medial arterial calcification (MAC) seen outlining the deep plantar arteries on a radiograph of the feet same evaluation without examiner knowledge of clinical or diabetic status. The examination included measurements of fasting serum cholesterol, urine protein, serum and urine creatinine, a 12-lead resting electrocardiogram, supine blood pressure determination, direct ophthalmoscopic examination by a physician after pupillary dilation, and a modified glucose tolerance test (GTT) in which glucose concentration was determined in plasma drawn $2 \mathrm{~h}$ after the ingestion of $75 \mathrm{~g}$ of carbohydrate (Dexcola, Custom Laboratories, Baltimore, Md; or Glucola, Ames Co., Elkhart, Ind, USA). Diabetes was diagnosed by a $2 \mathrm{~h}$ post-load plasma glucose concentration of at least $11.1 \mathrm{mmol} / \mathrm{l}$. Body mass index, a measure of obesity, was calculated as weight $/$ height $^{2}$ in $\mathrm{kg} / \mathrm{m}^{2}$.

Study participants age 15 years and older had posteroanterior radiographs of the hands and and feet and soft tissue radiographs of the lateral left calf and anteroposterior left thigh performed every 2 years until 1972, and then every 4 years. Pelvic radiographs were performed every 4 years in women age 45 years and older and men age 15 years and older. All radiographs were performed regardless of known disease and were examined for arterial calcification by one of the authors who had no knowledge of the clinical data. Linear calcification was categorised as MAC and any patchy calcification was classified as intimal calcification [2]. Of the 14,690 radiographs evaluated for calcification, $33(0.24 \%)$ had intimal calcification, $4(0.03 \%)$ had both intimal and medial calcification, and in $36(0.25 \%)$ the type of calcification could not be easily determined and was classified as indeterminant. For the purposes of this report, radiographs with both intimal and medial calcification were categorised as MAC while radiographs that were indeterminant or only found to have intimal calcification were categorised as not having MAC. The group with intimal calcification was considered too small for separate analysis.

Urine was obtained at the beginning of the GTT and discarded. Presence of protein was determined by Labstix (Ames Co.) on another sample obtained at the end of the GTT. For those samples containing at least $1+$ protein, a quantitative determination of proteinuria was made [15]. Heavy proteinuria was defined as a urine protein $(\mathrm{g} / \mathrm{l})$ to urine creatinine $(\mu \mathrm{mol} / \mathrm{l})$ ratio of greater than $1.13 \times 10^{-4} \mathrm{~g} /$ $\mu \mathrm{mol}$, which corresponds to a protein excretion of approximately $1 \mathrm{~g}$ or more per 24-h period [16,17]. Diabetic retinopathy, determined by direct ophthalmoscopy, was diagnosed by the presence of one or more microaneurysms or haemorrhages or of proliferative retinopathy. Electrocardiograms were coded according to Minnesota code
(MC) [18]. Electrocardiographic abnormalities considered indicative of coronary artery disease were $\mathrm{Q}$ waves (MC I-1, I-2), left bundle branch block (MC VIII-1), and complete heart block (not observed). From 1965 until 1978, vibration perception threshold of the left great toe was measured with a biothesiometer (Bio-Medical Instrument Co., Chagrin Falls, Ohio, USA), which provides $1-50$ volts to a vibrator. All patients who had lower extremity amputations for nontraumatic conditions between 1 July 1972 and 30 September 1982 were identified by review of medical records at the Phoenix Indian Medical Center, which is the general and orthopaedic surgical referral hospital for this population.

\section{Statistical analysis}

The prevalence of MAC was the percentage of subjects with MAC at the last examination in which a radiograph was taken. Prevalence rates were computed for 1381 diabetic patients and 3,172 non-diabetic subjects who had at least one radiographic examination. The incidence of MAC, computed in subjects who did not have MAC at their first examination, was the number of new cases divided by the time at risk according to diabetes status. Time until diagnosis of diabetes or until MAC was detected was tabulated as nondiabetic time at risk for MAC. Time from the diagnosis of diabetes until the detection of MAC was tabulated as diabetic time at risk for MAC. Incidence rates were expressed as cases per thousand person-years at risk for the 3065 participants without MAC on their first examination who had at least one subsequent examination. Age and sex adjusted incidence rate ratios for MAC were computed by the Mantel and Haenszel procedure modified for person-time denominators [19, 20]. A similar method was used to determine the incidence rate ratio for the diagnosis of diabetes according to the presence or absence of MAC, although cases were excluded in which MAC and diabetes were discovered at the same examination.

Proportional hazards survival analysis modelling was used to examine peripheral neuropathy as a risk factor for MAC and MAC as a risk factor for proteinuria, amputations, retinopathy, coronary artery disease, and mortality controlling for potentially confounding factors [21]. The hazard rate ratio for a risk factor was defined as the incidence of an event occurring at a given time for individuals with a particular risk factor divided by the incidence of the event occurring at the same time for individuals without that risk factor and is equiv- 


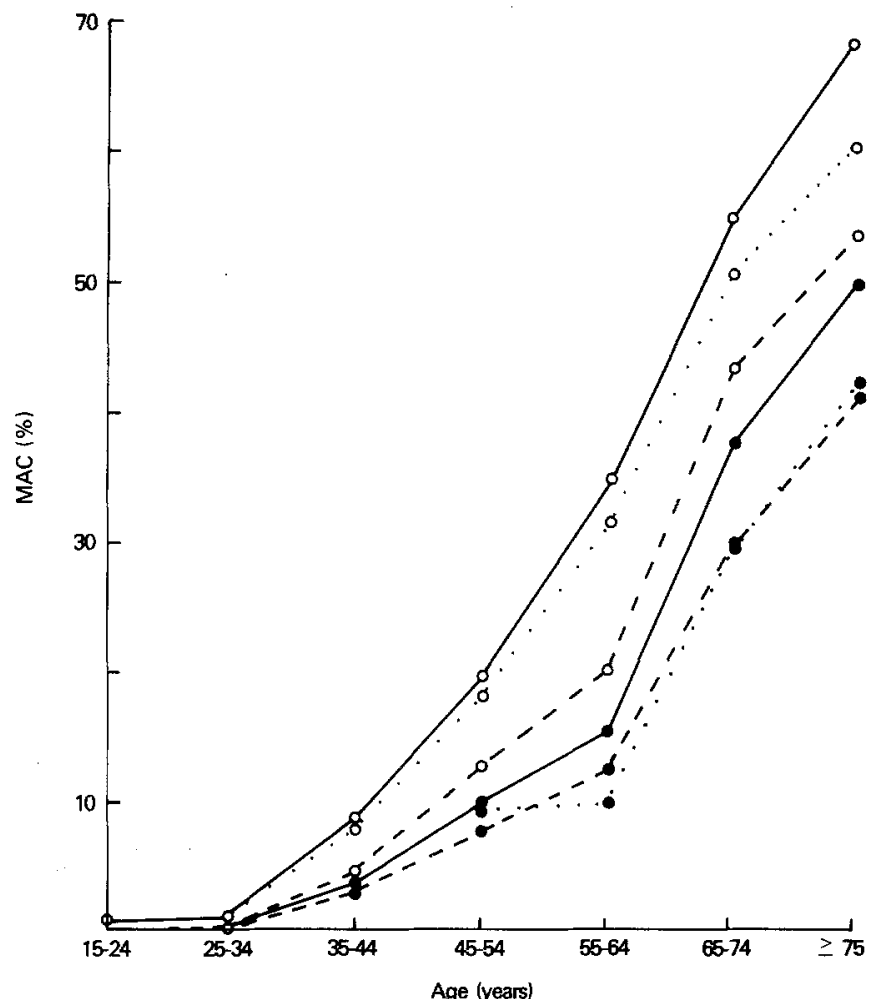

Fig. 2. Prevalence of medial arterial calcification (MAC) in \% in

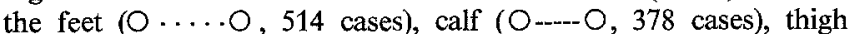
(- 324 cases), hands (---- 262 cases) and pelvis (-.... 127 cases) according to age at last radiographic examination. Prevalence for pelvis was truncated below 45 years of age as pelvic radiographs on women were not performed earlier than this age. $\mathrm{O}-\mathrm{O}$ A sites

Table 1. Age-adjusted prevalence (\%) of medial arterial calcification by radiographic site. Because pelvic radiographs were not performed in women under age 45 years, age-adjusted prevalence is not shown for this group

\begin{tabular}{|c|c|c|c|c|}
\hline \multirow{2}{*}{$\begin{array}{l}\text { Radiographic } \\
\text { site }\end{array}$} & \multicolumn{2}{|l|}{ Men } & \multicolumn{2}{|l|}{ Women } \\
\hline & $\begin{array}{l}\text { Non- } \\
\text { diabetic }\end{array}$ & Diabetic & $\begin{array}{l}\text { Non- } \\
\text { diabetic }\end{array}$ & Diabetic \\
\hline Any site & 24.7 & 39.5 & 10.7 & 27.7 \\
\hline Feet & 20.8 & 37.9 & 9.3 & 24.6 \\
\hline Calf & 17.1 & 28.9 & 7.7 & 19.4 \\
\hline Thigh & 15.6 & 25.7 & 4.4 & 15.7 \\
\hline Pelvis & 12.5 & 23.4 & - & - \\
\hline Hands & 8.8 & 22.9 & 5.3 & 14.1 \\
\hline
\end{tabular}

Table 2. Site of medial arterial calcification (MAC) in the lower extremity. Cases in whom MAC was concurrently detected in more than one site were excluded

\begin{tabular}{|c|c|c|c|c|c|}
\hline & Feet & \multicolumn{2}{|c|}{ Calf } & \multicolumn{2}{|c|}{ Thigh } \\
\hline & $\% \quad(n)$ & $\%$ & $(n)$ & $\%$ & $(n)$ \\
\hline Site first observed & $83 \quad(218)$ & 12 & (32) & 5 & (13) \\
\hline $\begin{array}{l}\text { Site next observed } \\
\text { after the feet }\end{array}$ & - & 82 & (23) & 18 & (5) \\
\hline
\end{tabular}

alent to the incidence rate ratio. The proportional hazards model assumes that the hazard rate ratio is constant during the course of a study, an assumption that appeared to be met by each variable in each model. There were no significant interactions of neuropathy or MAC with other variables, including age and sex.

To determine predictors of MAC, at least two radiographic examinations were required with MAC being absent on the first. Age and sex adjusted hazard rate ratios were initially determined for potential risk factors for MAC. Those factors whose $95 \%$ confidence interval did not include $1(p<0.05)$ were evaluated together with age and sex.

For diabetic patients, the effects of MAC on subsequent diabetic complications and mortality were determined using proportional hazards analysis. The presence of MAC and values of other variables were determined at the last examination within five years of the diagnosis of diabetes. At this baseline examination, 913 diabetic patients had all measurements and were thus eligible for the subsequent study of mortality. Time at risk in these analyses was the duration of known diabetes up to the study end point. For mortality, the end point was date of death or 1 October 1985 for those alive on that date. The study end point for amputations was the date of first amputation or 1 July 1982 for those who had not an amputation by that date. For heavy proteinuria, retinopathy, and coronary artery disease, the end point was the date at which the abnormality was first noted or the date of the last examination if the abnormality was not observed. Occurrences of death and amputations, heavy proteinuria, retinopathy, and coronary artery disease were analysed only if they were first documented at least 5 years after diabetes diagnosis. There were fewer participants evaluated for outcomes other than mortality primarily because of missing data such as vibration perception threshold for amputation and incomplete follow-up for retinopathy, proteinuria, and coronary artery disease.

\section{Results}

The prevalence of MAC at last examination is shown in Figure 2 for all 4553 study participants at their last examination according to age and site of radiographic examination. For each radiographic site, the prevalence of MAC was highest in older age groups, The prevalence of MAC was highest in distal sites of the lower extremity, so that age specific prevalence rates were greatest in the feet and least in the pelvis and hands. The prevalence of MAC, age-adjusted to a uniformly distributed population, is shown in Table 1 for the various radiographic sites according to sex and diabetic status. For each radiographic site, diabetic men and women had a higher age adjusted prevalence of MAC than their non-diabetic counterparts $(p<0.01)$. The greater prevalence of MAC in the feet suggests that MAC may begin distally. This possibility is supported by Table 2, which shows the first and second detected sites of MAC for persons with concurrent radiographs of the feet, calf, and thigh. Of those with MAC present at only one site, the feet were the site of calcification in $83 \%$ of cases ( $86 \%$ of the non-diabetic subjects and $82 \%$ of the diabetic patients). Of those with MAC only in the feet who subsequently developed MAC elsewhere, it was observed next in the calf in $82 \%$.

Figure 3 shows that the incidence of MAC in the feet was higher among diabetic patients than non-diabetic subjects. Similar relationships of age, sex, and 

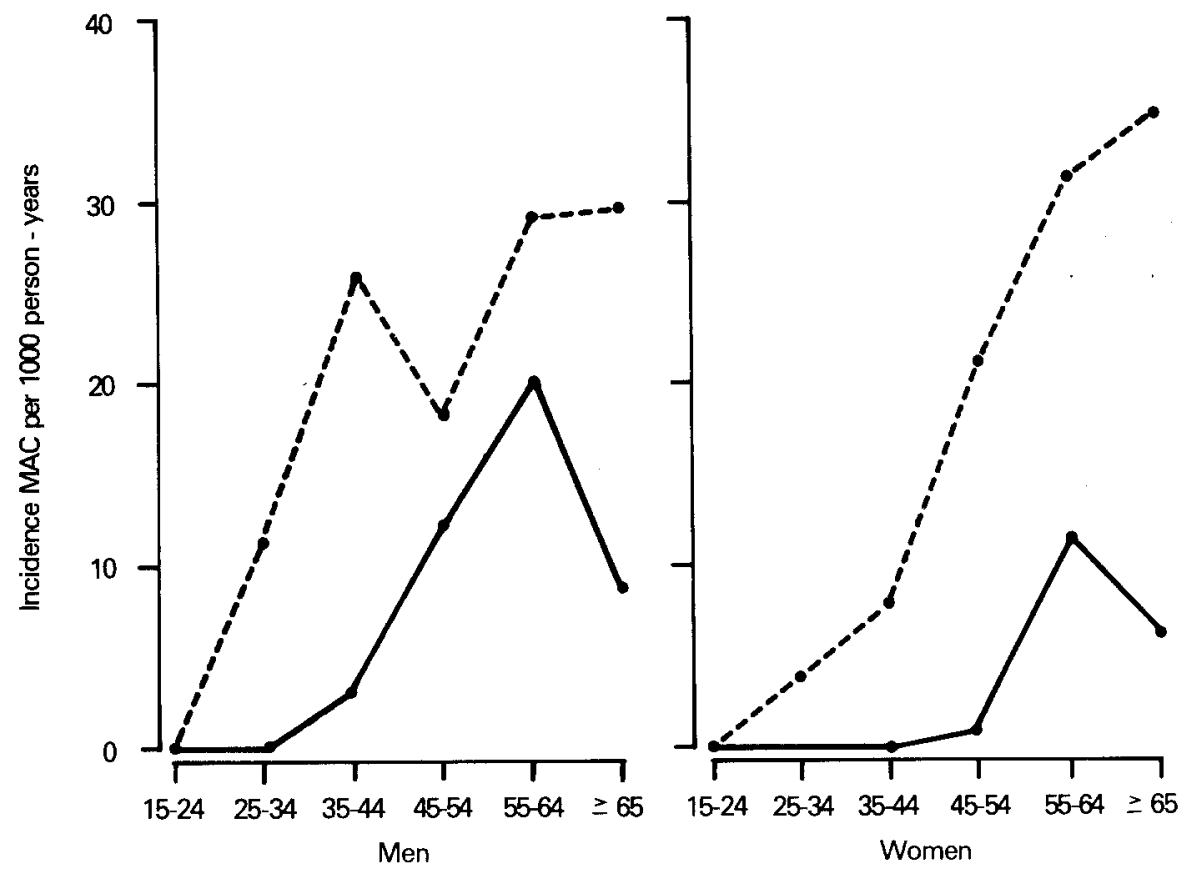

Fig.3. Incidence of medial arterial calcification (MAC) in the feet in cases per 1,000 person-years of observation according to age, sex, and presence of diabetes. There were 149 incident cases of MAC in 6,272 person-years among diabetic patients (-...) and 29 cases in 29,594 person-years among non-diabetic subjects $(-)$ ). The age-adjusted diabetic to non-diabetic incidence rate ratio and $95 \%$ confidence interval for men was 3.0 (1.8-5.1) and for women was 6.7 $(3.7-12.2)$

Age (years)

Table 3. Age and sex adjusted rate ratios for development of medial arterial calcification (MAC) of the feet for potential risk factors among 640 diabetic patients and 1223 non-diabetic subjects without MAC at the time of first examination. During follow-up 149 diabetic and 29 nondiabetic subjects developed MAC

\begin{tabular}{|c|c|c|c|c|c|}
\hline & & \multicolumn{2}{|c|}{ Diabetic patients } & \multicolumn{2}{|c|}{ Non-diabetic subjects } \\
\hline & & \multicolumn{2}{|c|}{ Rate confidence } & \multicolumn{2}{|c|}{ Rate confidence } \\
\hline Vibration perception threshold (V) & $\begin{array}{l}10-20 /<10 \\
\geq 20 /<10\end{array}$ & $\begin{array}{l}2.2 \\
2.3\end{array}$ & $\begin{array}{l}1.5-3.2 \\
1.3-4.1\end{array}$ & $\begin{array}{l}0.91 \\
0.58\end{array}$ & $\begin{array}{l}0.34-2.4 \\
0.15-2.2\end{array}$ \\
\hline Systolic blood pressure (mm $\mathrm{Hg}$ ) & $\begin{array}{l}120-165 / \leq 120 \\
\geq 165 / \leq 120\end{array}$ & $\begin{array}{l}1.3 \\
1.3\end{array}$ & $\begin{array}{l}0.78-2.2 \\
0.61-2.7\end{array}$ & $\begin{array}{l}0.53 \\
0.63\end{array}$ & $\begin{array}{l}0.22-1.3 \\
0.10-3.8\end{array}$ \\
\hline Diastolic blood pressure (mm $\mathrm{Hg}$ ) & $\begin{array}{l}80-105 /<80 \\
\geq 105 /<80\end{array}$ & $\begin{array}{l}0.98 \\
0.58\end{array}$ & $\begin{array}{l}0.70-1.4 \\
0.21-1.6\end{array}$ & $\begin{array}{l}0.82 \\
6.3\end{array}$ & $\begin{array}{l}0.27-1.8 \\
1.3-30.3\end{array}$ \\
\hline Serum cholesterol $(\mathrm{mmol} / \mathrm{l})$ & $\begin{array}{l}4.66-6.46 /<4.66 \\
\geq 6.47 / 4.66\end{array}$ & $\begin{array}{l}1.1 \\
1.2\end{array}$ & $\begin{array}{l}0.80-1.6 \\
0.68-2.2\end{array}$ & $\begin{array}{l}2.2 \\
4.5\end{array}$ & $\begin{array}{l}0.96-5.1 \\
1.3-15.3\end{array}$ \\
\hline Ankle reflexes & absent/present & 2.2 & $1.5-3.1$ & 1.1 & $0.43-2.6$ \\
\hline Serum creatinine $(\mu \mathrm{mol} / 1)$ & $\begin{array}{l}88.4-133 /<88.4 \\
>133 /<88.4\end{array}$ & $\begin{array}{l}2.0 \\
0.69\end{array}$ & $\begin{array}{l}1.0-3.7 \\
0.28-1.7\end{array}$ & $\begin{array}{l}0.23 \\
2.9\end{array}$ & $\begin{array}{l}0.03-1.8 \\
0.85-9.7\end{array}$ \\
\hline Proteinuria & $>$ trace/none & 1.1 & $0.64-2.0$ & 0.52 & $0.07-4.0$ \\
\hline Diabetes duration (years) & $\begin{array}{l}1-4 / \leq 1 \\
>4 / \leq 1\end{array}$ & $\begin{array}{l}2.1 \\
4.0\end{array}$ & $\begin{array}{ll}1.4 & -3.1 \\
2.7 & -5.9\end{array}$ & $\begin{array}{l}- \\
-\end{array}$ & $\begin{array}{l}- \\
-\end{array}$ \\
\hline
\end{tabular}

diabetes were found at proximal sites and the hands, but with lower incidence rates of MAC (not shown). The age adjusted male to female incidence rate ratio of MAC of the feet for nondiabetic subjects was $3.0(95 \%$ confidence interval $=1.4$ to 6.3 ) and for diabetic patients $1.3(95 \%$ confidence interval $=0.87$ to 1.9$)$.
The incidence of diabetes according to presence of MAC of the feet was also evaluated. There were 443 persons who developed diabetes before MAC was detected and only 25 who developed diabetes after MAC was detected. The incidence of diabetes for persons with MAC of the feet compared to persons with- 


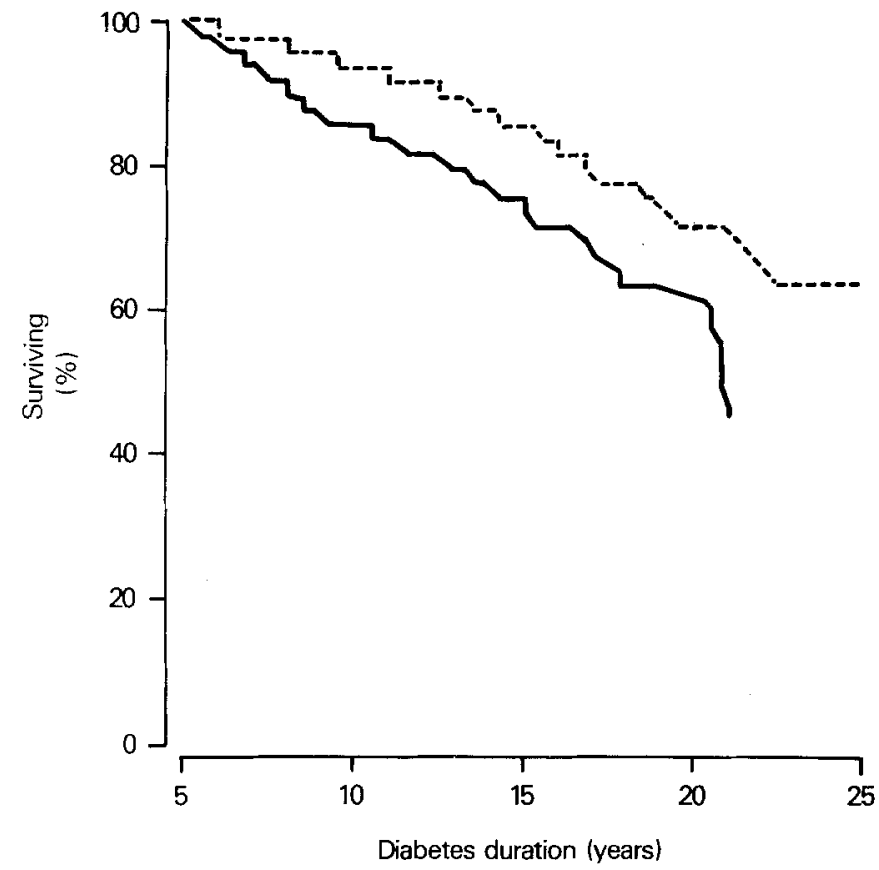

Fig.4. Estimated survival curve for mortality according to the presence (-) or absence (----) of medial arterial calcification (MAC) at an examination within 5 years of the diagnosis of diabetes controlling for variables noted in Table 4

out MAC of the feet was $0.53(95 \%$ confidence inter$\mathrm{val}=0.33$ to 0.83 ).

As the feet were the most frequent and usually the earliest site of MAC, further results for risk factors and consequences of MAC focus on radiographs of this site. In general, evaluation of MAC at other sites led to similar conclusions. Age and sex adjusted rate ratios for levels of potential risk factors are shown stratified by diabetic status in Table 3 . Participants could contribute time at risk to both analyses if they did not have diabetes at first examination and subsequently developed diabetes prior to the detection of MAC. There was a significantly higher $(p<0.05)$ age and sex adjusted risk of MAC for those diabetic patients with high vibration perception thresholds, highest tertile of 2-h post-load plasma glucose concentrations, absent ankle reflexes, the mid-range of serum creatinine concentration, or longer duration of diabetes.

Of particular interest was the relationship of peripheral neuropathy as measured by vibration perception threshold to the development of MAC in the feet. In this study, $20 \mathrm{~V}$, which was the 95th percentile of vibration perception threshold among the non-diabetic subjects, was chosen arbitrarily as an upper normal limit. The effect of neuropathy on MAC was calculated as the ratio of the hazard rates for development of MAC for those with abnormally high vibration perception threshold and those with a threshold of less than $10 \mathrm{~V}$. Diabetic patients with abnormal vibration perception developed MAC of the feet at 2.3-fold the rate (95\% confidence interval $=1.4$ to $3.9, p<0.01$ ) of dia- betic patients with vibration perception of less than 10 volts when controlling for age, sex, and the other significant factors from the age-sex adjusted analysis with proportional hazards analysis. Of the other variables analysed, degree of hyperglycaemia and duration of diabetes $(p<0.01$ for each variable) were also associated with development of MAC in the proportional hazards model, while age, sex, serum creatinine concentration, and absent ankle reflexes were not.

MAC in the feet was detected in 29 of the 1,223 non-diabetic subjects following an initial negative examination. Controlling for age and sex, the detection rate of MAC was greater for subjects with high serum cholesterol concentrations or high diastolic blood pressures (Table 3). These variables, age, sex, and vibration perception threshold were evaluated together as risk factors for MAC. There was no relationship of impaired vibration perception threshold to the development of MAC (hazard rate ratio $=1.3$ with $95 \%$ confidence interval $=0.37$ to 4.3 ), but age and male gender were predictive of MAC $(p<0.01)$ as was a high serum cholesterol concentration $(p=0.02)$.

The presence or absence of MAC in the feet was ascertained at the last examination in which a radiograph was obtained within 5 years of the diagnosis of diabetes. Rates of mortality and subsequent diabetic complications were determined after a median duration of follow-up of 12.4 years after diabetes diagnosis. As shown in Table 3 and Figure 4, the mortality rate was higher for diabetic patients with MAC than without MAC even when controlling for age, sex, degree of hyperglycaemia, blood pressure, serum cholesterol concentration, presence or absence of proteinuria, and body mass index (hazard rate ratio $=1.5$ with $95 \%$ confidence interval $=1.0$ to 2.1 ). Controlling only for age and sex, the mortality hazard ratio for diabetic patients was $1.9(95 \%$ confidence interval $=1.3$ to 2.3$)$. Among non-diabetic subjects, controlling for age and sex, those with MAC did not have a higher mortality rate than those without MAC (incidence rate ratio $=0.95$, $95 \%$ confidence interval $=0.70$ to 1.3 ).

Of the 36 first time amputations performed among the diabetic patients during the study period, $28(78 \%)$ were of toes. The presence of MAC was associated with a marked increase in risk of amputation (unadjusted hazard rate ratio $=5.6,95 \%$ confidence inter$\mathrm{val}=2.9$ to 10.7). This association persisted even when controlling for impaired vibration perception and absent ankle jerks (Table 4, Fig.5). Controlling only for age and sex the rate of amputations among diabetic smokers was 1.1-fold that of diabetic non-smokers (95\% confidence interval $=0.79$ to 1.6$)$. Smoking was not included in the multivariate model of amputation because smoking history was not gathered initially in the study and the proportion of smokers is low among the Pima Indians, with only $0.6 \%$ of study participants reporting cigarette smoking of at least a pack per day.

Risk of development of proteinuria and retinopa- 
Table 4. Rate ratios for mortality and diabetic complications according to presence or absence of medial arterial calcification (MAC) within 5 years of diabetes diagnosis

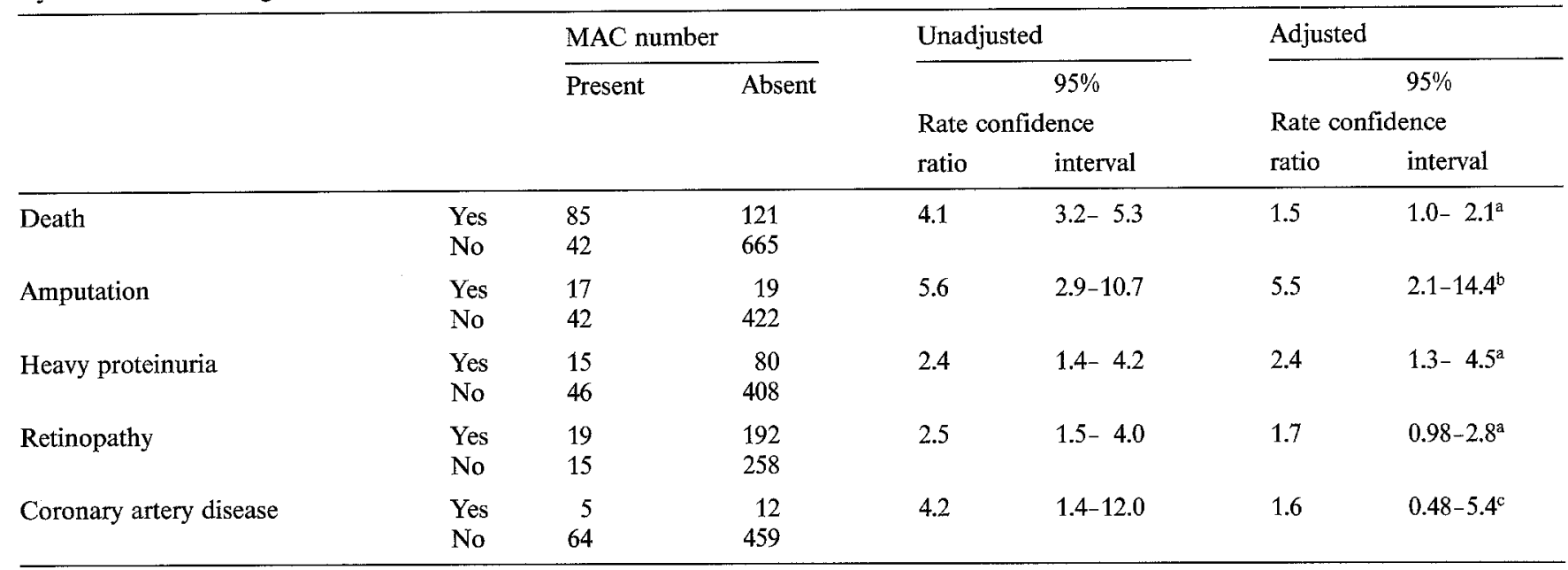

${ }^{a}$ Proportional hazards analysis controlling for age, sex, 2-h post-load plasma glucose, blood pressure serum cholesterol, proteinuria, and body mass index at examination in which MAC was determined. ${ }^{\mathrm{b}}$ Proportional hazards analysis controlling for age, sex, 2 -h post-load plasma glucose, blood pressure, serum cholesterol, proteinuria, body mass index, vibration perception threshold, and ankle reflexes at examination in which MAC was determined. ${ }^{\circ}$ Proportional hazards analysis controlling for age and sex at examination in which MAC was determined

thy among diabetic patients with MAC was greater than for those without MAC, but the difference in risk were not as pronounced as for amputation (Table 4, Fig.5). There were only 17 cases of electrocardiographic changes indicative of coronary artery disease. The hazard rate ratio of 1.6 for coronary artery disease was calculated controlling only for age and sex and had a wide $95 \%$ confidence interval of 0.48 to 5.4 (Table 4). Similar increased risks of mortality and diabetic complications were found for patients with MAC at radiographic sites other than the feet (data not shown).

\section{Discussion}

MAC occurs more commonly in diabetic than non-diabetic Pima Indians. The greatest prevalence of MAC was in elderly diabetic men. Similar relationships have been found in other studies involving large numbers of participants $[8,22]$. In our study, the incidence of MAC in the feet of non-diabetic men was 3.0-fold that of non-diabetic women, but among diabetic men it was only 1.3 -fold that of diabetic women. The similar rates of MAC among diabetic men and women but not nondiabetic men and women is a pattern seen with other diabetic vascular complications, such as ischaemic heart disease [23-25] and peripheral vascular disease [26].

The observation that MAC was usually found first in the feet and then the calf indicates that the lesion originates in the smaller or more distal leg arteries and progesses proximally. It is possible, however, that MAC may be detected more easily in these distal sites, leading to higher distal prevalence and incidence estimates. A careful study that compared plain radio-
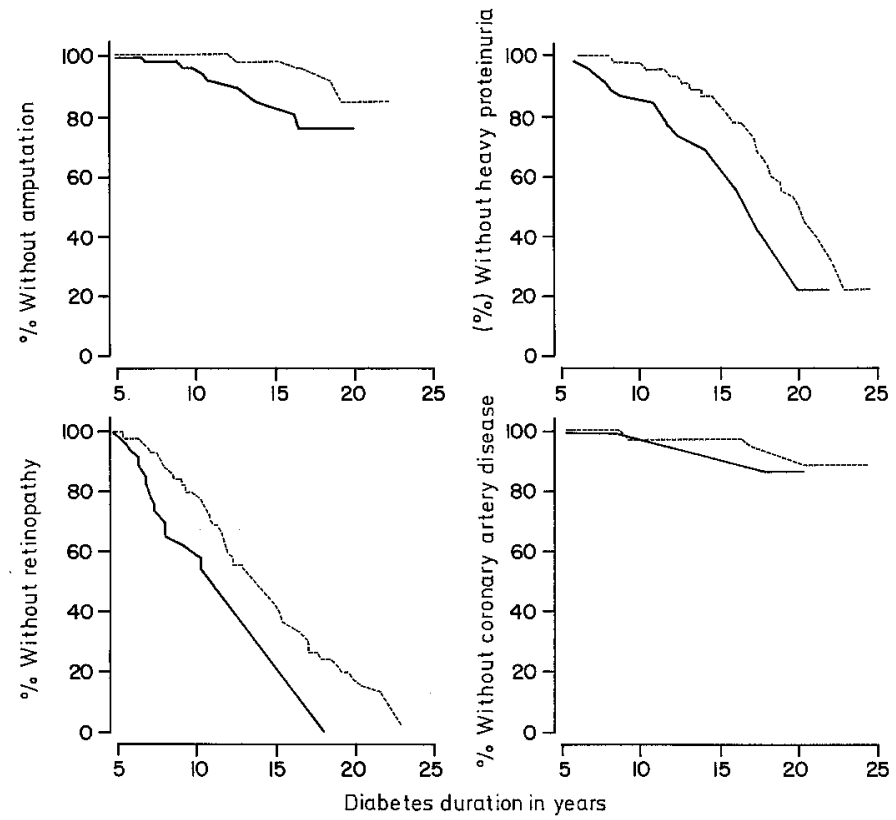

Fig.5. Estimated survival curves for amputation, heavy proteinuria, retinopathy, and coronary artery disease according to the presence (-) or absence (---) of medial arterial calcification (MAC) at an examination within 5 years of the diagnosis of diabetes controlling for variables noted in Table 4

graphs to angiography did not comment on a low likelihood of proximal detection of MAC [2]. More frequent evaluation with measurement of extension of MAC would be necessary to confirm proximal progression.

Most participants developed diabetes before MAC was detected. Yet it is not clear why subjects with MAC would have a lower incidence of diabetes than subjects without MAC. It is possible that MAC devel- 
oped before diabetes was diagnosed in some cases that were excluded from analysis because MAC and diabetes were detected simultaneously.

A possible causal relationship between neuropathy and MAC is suggested by the increased risk of MAC in diabetic patients with previously impaired perception of vibration. This effect of impaired sensation persisted after controlling for age, sex, and other risk factors for peripheral vascular disease. Other studies have also implicated neuropathy as a cause of MAC. Deterioration of arterial smooth muscle cells has been demonstrated following sympathetic denervation in rabbits $[4,5]$. Human studies have shown that MAC in associated with diabetic peripheral neuropathy [6] and familial amyloidosis with polyneuropathy [11], and occurs in the ipsilateral leg following lumbar sympathectomy [7]. In the University Group Diabetes Program, however, the relationship of vibration perception threshold to calcification was not significant in either sex, but systolic blood pressure and serum triglyceride concentration were associated with development of arterial calcification in diabetic women [12]. That report did not discriminate medial from intimal calcification, which may have obscured a relationship of neuropathy to MAC.

Among non-diabetic subjects, neuropathy did not predict subsequent MAC, but older age, higher serum cholesterol concentrations, and being male were predictive. While there have been no previous reports of an association of serum cholesterol concentration and MAC, both elevated serum cholesterol concentrations and diabetes have been associated with calcific aortic stenosis, another vascular calcific disorder of obscure cause [27]. It is unknown if MAC and calcific aortic stenosis are otherwise related.

Higher all-cause mortality among diabetic patients with MAC but not non-diabetic subjects with MAC might be explained by the association of MAC and subsequent amputations, proteinuria and retinopathy, which occurred almost exclusively among diabetic patients. The association of MAC with amputation was particularly marked, as the rate of first amputation for diabetic patients with MAC was 5.5-fold that of patients without MAC. There may be anatomical and physiological reasons why MAC predicts amputation. A positive relationship of MAC with narrowing and obstruction of the arteries was reported in feet amputated for peripheral vascular disease and gangrene [28]. Blood flow in the legs has been found to be lower for diabetic patients with MAC than for those without MAC $[29,30]$. A study of diabetic gangrene and amputations did not find a significantly higher rate of MAC in patients with gangrene, but did find that the presence of MAC among patients with gangrene was positively related to subsequent amputation [11].

Reasons for the association of MAC in the feet and subsequent proteinuria and retinopathy are not as clear as for the association of MAC and amputations.
Peripheral neuropathy, which predicts the development of MAC, is also associated with proteinuria and retinopathy, even when controlling for degree of hyperglycaemia [31]. Thus MAC may be an early manifestation of a metabolic, vascular, or other process which precedes the onset of overt proteinuria and retinopathy. Because of the few cases of electrocardiographic changes suggestive of myocardial infarction, it is difficult to draw firm conclusions concerning MAC and the risk of coronary artery disease.

MAC is predictive of diabetic complications and mortality and thus is of prognostic significance in Type 2 diabetes. Recognition of the presence of MAC may help identify individuals who are at high risk of diabetic complications, particularly amputation, and who, therefore, might benefit from intensive foot-care and other types of treatment.

Acknowledgements. The authors are indebted to the members of the Gila River Indian Community who participated in this investigation; the technical, clerical and physician staff of the Diabetes and Arthritis Epidemiology Section, NIDDK for conducting examinations and gathering data; Dr. D. Gohdes of the Indian Health Service for collection of data on amputations; and Dr. I. Liebow for interpretation of electrocardiograms.

\section{References}

1. Lachman AS, Spray TL, Kerwin DM, Shugell GI, Roberts WC (1979) Medial calcinosis of Mönckeberg. A review of the problem and a description of a patient with involvement of peripheral, visceral and coronary arteries. Am J Med 63: 615-622

2. Lindbom $\AA$ (1950) Arteriosclerosis and arterial thrombosis in the lower limb. A roentgenological study. Acta Radiol [Suppl] 80: $1-80$

3. Bierman EL (1983) Atherosclerosis and other forms of arteriosclerosis In: Petersdorf RG, Adams RD, Braunwald E, Isselbacher KJ, Martin JB, Wilson JD (eds) Harrison's principles of internal medicine, 10th edn. McGraw-Hill, New York, pp 1465-1475

4. Bevan RD (1975) Effect of sympathetic denervation on smooth muscle cell proliferation in the growing rabbit ear. Circ Res 37 : 14-19

5. Bevan RD, Tsuru H (1979) Long-term denervation of vascular smooth muscle causes not only functional but structural change. Blood Vessels 16: 109-112

6. Edmonds ME, Morrison N, Laws JW, Watkins PJ (1982) Medial arterial calcification and diabetic neuropathy. Br Med J 284: 928-930

7. Goebel F-D, Füssl HS (1983) Mönckeberg's sclerosis after sympathetic denervation in diabetic and non-diabetic subjects. Diabetologia $24: 347-350$

8. Ferrier TM (1964) Radiologically demonstrable arterial calcification in diabetes mellitus. Aust Ann Med 13: 222-228

9. Parfitt AM (1969) Soft-tissue calcification in uremia. Arch Intern Med 124: 544-555

10. Neubauer B (1971) A quantitative study of peripheral arterial calcification and glucose tolerance in elderly diabetics and non-diabetics. Diabetologia 7: 409-13

11. Lithner F, Hietala S-O, Steen L (1984) Skeletal lesions and arterial calcifications of the feet in diabetics. Acta Med Scand [Suppl] 687: 47-54

12. Kreines $\mathrm{K}$, Johnson E, Albrink M, Knatterud G, Levin M, Lewi$\tan$ A, Newberry W, Rose F (1985) The course of peripheral vas- 
cular disease in non-insulin-dependent diabetes. Diabetes Care $8: 235-243$

13. Nilsson SE, Lindholm H, Bülow $\mathrm{S}$, Frostberg N, Emilsson T, Stenkula G (1964) The Kristianstad survey 63-64. Acta Med Scand [Suppl] 428: 1-46

14. Knowler WC, Bennett PH, Hamman RF, Miller M (1978) Diabetes incidence and prevalence in Pima Indians: 19-fold greater incidence than in Rochester, Minnesota. Am J Epidemiol 108: 497-505

15. Shevky MC, Stafford DD (1923) A clinical method for the estimation of protein in urine and other body fluids. Arch Intern Med 32: 222-225

16. Ginsberg JM, Chang BS, Matarese RA, Garella S (1983) Use of single voided urine samples to estimate quantitative proteinuria. N Engl J Med 309: 1543-1546

17. Shaw AB, Risdon P, Lewis-Jackson JD (1983) Protein creatinine index and Albustix in assessment of proteinuria. Br Med $\mathrm{J} 287$ : 929-932

18. Blackburn $H$ (1969) Classification of the electrocardiogram for population studies: Minnesota code. J Electrocardiol 2: 305-310

19. Mantel N, Haenszel W (1959) Statistical aspects of the analysis of data from retrospective studies of disease. J Natl Cancer Inst 22: 719-748

20. Rothman KJ, Boice JD (1982) Follow-up (cohort) studies. In: Epidemiologic analysis with a programmable calculator. Epidemiology Resources, Boston, pp 11-17

21. Harrell FE (1983) The PHGLM procedure. In: Joyner SP (ed) SUGI supplemental library user's guide, 1983 edn. SAS Institute, Cary, North Carolina, pp 267-94

22. Nilsson SE, Nilsson JE, Frostberg N, Emilsson T (1967) The Kristianstad survey II. Acta Med Scand [Suppl] 469: 1-42

23. Heyden S, Heiss G, Bartel AG, Hanes CG (1980) Sex differences in coronary mortality among diabetics in Evans county, Georgia. J Chronic Dis 33: 265-273

24. Barrett-Connor E, Wingard DL (1983) Sex differential in ischem- ic heart disease mortality in diabetics. Am J Epidemiol 118: 489-496

25. Butler WJ, Ostrander LD, Carman WJ, Lamphear ED (1985) Mortality from coronary heart disease in the Tecumseh study. Am J Epidemiol 121: 541-547

26. Kannel WB, McGee L (1979) Diabetes and cardiovascular disease. The Framingham study. JAMA 241: 2035-2038

27. Deutscher S, Rockette HE, Krishnaswami V (1984) Diabetes and hypercholesterolemia among patients with calcific aortic stenosis. J Chron Dis 37: 407-415

28. Ferrier TM (1967) Comparative study of arterial disease in amputated lower limbs from diabetics and non-diabetics (with special reference to feet arteries). Med J Austr 1: 5-11

29. Christensen NJ (1968) Muscle blood flow, measured by xenon ${ }^{133}$ and vascular calcifications in diabetics. Acta Med Scand 183: 449-454

30. Christensen NJ (1973) Diabetic macroangiopathy: blood flow and radiological studies. In: Camerini-Davalos RA, Cole HS (eds) Vascular and neurological changes in early diabetes. Academic, New York, pp 129-135

31. Young RJ, Macintyre CCA, Martyn CN, Prescott RJ, Smith AF, Viberti G, Clarke BF (1986) Progression of subclinical polyneuropathy in young patients with Type 1 (insulin-dependent) diabetes: associations with glycaemic control and microangiopathy (microvascular complications). Diabetologia 29: 156-161

Received: 16 June 1987

and in revised form: 30 October 1987

Dr. JE Everhart

Federal Building, Room 106

7550 Wisconsin Avenue

Bethesda, MD 20892

USA 